28 PAEDIATRIC MENTAL HEALTH PROVISION DURING COVID - A FOOTPRINT FOR THE FUTURE?

Carly Vassar, John Forrester. Great Ormond Street Hospital

10.1136/archdischild-2020-gosh.28

During the first surge of the covid pandemic paediatric services across North London had to rapidly adapt to a system urgently needing to increase adult capacity, in response general paediatric admissions including acute mental health presentations were diverted to Great Ormond Street Hospital. Prospective data on types of admissions were collated throughout the surge, identifying trends and allowing the rapid development of nursing services to ensure high quality and safe provision of care for an acute group of patients.

What we did? Data was collected to show demographics, length of stay, use of the mental health act, local hospital and discharge destination.

The complexity of patients seen required a rapid and evolving set up of a dedicated space to house this group of dedicated patients. Risk assessments of space, CQC registration and support through the local mental health trust needed rapid review and adjustments. Paediatric nursing staff who had not previously worked within mental health needed rapid and rolling training on topics around safety, the mental health act and the law.

Networks with local community services were rapidly stepped up and daily planning meetings evolved.

What we found? 48 patients were admitted, between 1 -9 days length of stay with an average of 3,9 were admitted under the mental health act.

Rapid response to an evolving need and changing picture with the backdrop of covid was a unique and challenging time. The rewards of this work, the relationships formed and the recognition from the wider sector around the high quality and therapeutic provision of care shaped direction and desire to ensure that this piece of work could be a footprint for the future modelling and development of mental health provision.

Upskilling paediatric nurses with concepts around therapeutic effect holds future potential for achieving truly holistic care regardless of setting.

\section{INVERTING THE WELLBEING PYRAMID: THE GOSH APPROACH TO LOOKING AFTER STAFF WELLBEING}

Mandy Bryon, Daljit Hothi, Kristina Soon. Great Ormond Street Hospital

\subsection{6/archdischild-2020-gosh.29}

At the start of the COVID-19 pandemic experts in trauma and psychology advised about the importance of ensuring the mental and emotional well-being of staff was being considered and supported. Most of the literature and experts advocated a layered, hierarchical intervention approach.

At Great Ormond Street Hospital (GOSH) we elected to 'invert the pyramid', mobilising the 'specialists/experts' to create a virtual well-being hub and that overtime layering and blending other interventions as suggested in this classical model.

One of the key successes of the COVID crisis at GOSH has been our ability to raise the profile and importance of staff wellbeing across the organisation and this has been well received.
The hub continues to remain the centre of our work and has been active identifying, triaging and providing psychological first aid or referring staff for external mental health treatment. In the recovery phase we have extended the 'expertise' to Peer Support Workers, TRiM Practitioners and Well-being Coaches that have collectively formed a pan-trust well-being network to meet the needs of the COVID-19 but also the smaller, repeated and significant traumas that we face when working in healthcare.

\section{DEVELOPMENT OF A MOBILE APP 'HEAR ME OUT' TO SUPPORT AUDIOLOGY PATIENTS}

${ }^{1}$ Max Bosch, ${ }^{1}$ Jason James, 'Lucy Rothwell, ${ }^{2}$ Daiana Bassi, ${ }^{2}$ Sue Conner, ${ }^{1}$ Yun Fu, ${ }^{1}$ Dean Mohamedally, ${ }^{1}$ Gemma Molyneux, ${ }^{1}$ Graham Roberts, ${ }^{1}$ Neil J Sebire, ${ }^{3}$ Raouf Chorbachi, ${ }^{3}$ Natalie Stephenson. ${ }^{1}$ UCL Department of Computer Sciences; ${ }^{2} D R I V E$, Great Ormond Street Hospital for Children NHS Foundation Trust; ${ }^{3}$ Audiology, Great Ormond Street NHS Foundation Trust

\subsection{6/archdischild-2020-gosh.30}

Transition into adult services can be a challenging time, where young patients are very quickly expected to take responsibility for their own medical care. Studies have shown that transition can be linked to a decline in health, as patients struggle to cope with the stresses and demands that go with the change from paediatric to adult services. In order to support children through transition, GOSH launched 'Growing Up, Gaining Independence' support programme. To further develop the support available through this programme, the Audiology team designed a mobile app aimed at patients preparing to transition into adult care.

As part of a joint collaboration between GOSH and UCL computer science (CS) through the industry exchange network, the prototype mobile app 'Hear Me Out' was developed using Ionic. In addition, a content management system was developed using NodeJS, with a MySQL database to allow content delivered via the app to be to edited and updated.

The mobile app provides helpful information to patients and families, including features such as 'MyStory' that allows patients to log important events such as appointments or symptoms. The app has support in the form of a glossary to help patients to understand medical terminology and includes information on NHS support services that provide further patient support. The app is ready to be trialled with patients and families, to gather feedback on how the design and content can be improved. This app is an example of how technology can be developed to support children and young people gain independence and manage their medical care with confidence.

\section{DEVELOPMENT OF A WEB-BASED SCREENING TOOL 'CRANIOQ' TO SUPPORT THE IDENTIFICATION OF CHILDREN WITH CRANIOFACIAL DEFECTS}

\footnotetext{
${ }^{1}$ Yasmin Abedin, ${ }^{2}$ Andrew Brinkmeyer, ${ }^{2}$ Sioban Hughes, ${ }^{3}$ Daiana Bassi, ${ }^{3}$ Sue Conner, ${ }^{2}$ Yun Fu, ${ }^{2}$ Dean Mohamedally, ${ }^{3}$ Gemma Molyneux, ${ }^{3}$ Neil J Sebire, ${ }^{4}$ Alessandro Borghi, ${ }^{5}$ Juling ONg. ' UC; ${ }^{2}$ UCL Department of Computer Sciences; ${ }^{3} D R I V E$, Great Ormond Street Hospital for Children NHS Foundation Trust; ${ }^{4} U C L$ Great Ormond Street Institute of Child Health; ${ }^{5}$ Craniofacial, Great Ormond Street NHS Foundation Trust
}

10.1136/archdischild-2020-gosh.31 